\title{
New set of microsatellite markers for the spotted-wing Drosophila suzukii (Diptera: Drosophilidae): A promising molecular tool for inferring the invasion history of this major insect pest
}

\author{
Antoine FRAIMOUT ${ }^{1}$, Anne LOISEAU ${ }^{2}$, Donald K. PRICE ${ }^{3}$, AnNe XUÉREB ${ }^{2}$, JeAn-François MARTIN ${ }^{2}$, \\ Renaud VITALIS ${ }^{2}$, Simon FELLOUS ${ }^{2, *}$, Vincent DEBAT $^{1, *}$ and ArNaUd ESTOUP ${ }^{2, *}$
}

${ }^{1}$ Institut de Systématique, Evolution et Biodiversité, ISYEB, UMR 7205, CNRS, UPMC, EPHE, Muséum National d'Histoire Naturelle, Sorbonne Universités, 45 rue Buffon, 75005 Paris, France; e-mails: antoine.fraimout@edu.mnhn.fr; debat@mnhn.fr

${ }^{2}$ INRA UMR, Centre de Biologie pour la Gestion des Populations (INRA/IRD/Cirad / Montpellier SupAgro),

755 avenue du Campus Agropolis, CS 30 016, 34988 Montferrier-sur-Lez cedex 17, France; e-mails: loiseau@supagro.inra.fr; xuereb@supagro.inra.fr; martinjf@supagro.inra.fr; vitalis@supagro.inra.fr; simon.fellous@supagro.inra.fr; estoup@supagro.inra.fr ${ }^{3}$ Department of Biology, and Tropical Conservation Biology and Environmental Science Graduate Program, University of Hawaii, 200 West Kawili Street, Hilo, HI 96720, USA; e-mail: donaldp@hawaii.edu

Key words. Diptera, Drosophilidae, Drosophila suzukii, population genetics, microsatellite, invasion routes, invasive species, genetic structure

\begin{abstract}
Historical and observational data for invasive species are often sparse and incomplete, so molecular genetic markers are increasingly used and have proved to be efficient tools to make inferences about invasion histories. Here, we report the development of 28 polymorphic microsatellite markers in the invasive spotted-wing drosophila, Drosophila suzukii (Matsumura, 1931) (Diptera: Drosophilidae) designed from recent genomics resources, and their cross-amplification in closely related Drosophila species of the suzukii subgroup. The markers, located on autosomal chromosomes, were characterized in two distinct invasive populations from France and Hawaii (USA), and in four sister species of the suzukii subgroup. They all showed substantial polymorphism as well as revealing strong genetic differentiation between the two genotyped populations. These markers represent a promising molecular tool for population genetic studies on D. suzukii, more especially in order to reconstruct the pathways and demographic processes of the world-wide invasion in this major insect pest.
\end{abstract}

\section{INTRODUCTION}

The spotted-wing drosophila, Drosophilla suzukii (Matsumura, 1931) (Diptera: Drosophilidae) is an oriental species of the melanogaster group. It is widely distributed in south-eastern Asia, from India (Parshad \& Paika, 1965) to Japan, where it was first described in 1931 (Hauser, 2011). D. suzukii females exhibit a large serrated ovipositor which allows them to lay eggs in ripening fruits, hence causing severe damages to stonefruit and red berry crops. The species has drawn particular attention during the past five years because of its status as a crop pest and its rapid invasion across Europe and the USA (reviewed in Hauser, 2011 and Calabria et al., 2012). When an invasive species presents immediate threats in a new environment (e.g. economical, ecological or for human health), most research plans legitimately focus on how to prevent further expansion or to reduce damages caused by introduced populations (Thresher et al., 2014). However, reconstructing the geographic pathways followed by propagules from their original source location to the newly invaded location (hereafter referred to as invasion routes) is often overlooked. In light of this, even though some crucial aspects of $D$. suzukii biology and ecology have been already investigated (Kimura, 2004), little is known about the species' invasion history and more especially the origins of the different introduced populations (but see Adrion et al., 2014 for a first approach). This is unfortunate, since robust information about the invasion routes of a species is crucial for at least two principle reasons: (i) it facilitates the design of strategies for controlling present or preventing future invasions; and (ii) it is requirement necessary for defining and testing different hypotheses concerning the environmental and evolutionary factors responsible for biological invasions in general (Estoup \& Guillemaud, 2010).

Most of our knowledge about the introduction routes of invasive species derives from historical and observational data, which are often sparse, incomplete and, sometimes, misleading. In the case of $D$. suzukii, this approach suggests there was a single introduction in Europe, located in France in between Spain and Italy, where the pest was first reported (Cini et al., 2014). An alternative approach is to use population genetics analyses based on polymorphic molecular markers, such as microsatellites, which have proved to be powerful tools for reconstructing invasion routes, highlighting the complexity and the often counter intuitive nature of the true story (Estoup et al., 2001; Estoup \& Guillemaud, 2010). Here we report the development of a set of 28 polymorphic microsatellite markers in D. suzukii designed from recent genomics resources, as well as characterizing genetic variation at these particular loci in two distinct invasive populations along with their cross-amplification patterns in closely related Drosophila species.

\section{MATERIAL AND METHODS}

\section{Sampling and DNA extraction}

Adult $D$. suzukii were collected from the field at two distinct localities of the invasive range between October and November, 2013, and stored in ethanol. The Hawaii population consisted of

\footnotetext{
* Equal authorship.
} 
33 adult flies caught at the Saddle Road locality (19 $47^{\prime} 35.1^{\prime \prime}$, $\left.155^{\circ} 30^{\prime} 54.3^{\prime \prime} \mathrm{W}\right)$, the French population consisted of 27 adult flies caught near Montpellier $\left(43^{\circ} 42^{\prime} 00^{\prime \prime} \mathrm{N}, 3^{\circ} 51^{\prime} 56^{\prime \prime} \mathrm{E}\right)$. Total genomic DNA was extracted using 10\% BioRad Chelex (Estoup et al., 1996).

\section{Primer design and PCR amplification}

We used the recently assembled $D$. suzukii genome (Chiu et al., 2013; project accession number PRJNA213258) as an input for the QDD program (Méglecz et al., 2014) to scan genomic data for microsatellite regions. Briefly, QDD uses four scripts (Sequence similarity, BLAST, microsatellite detection and markers design) to analyze raw data and provide a table summarizing, amongst other, the region of the genome containing a potential microsatellite sequence, the repeat motif and the number of repeats. We decided to further filter the results of QDD by choosing only continuous (i.e. non-interrupted) microsatellite sequences, with at least 10 repetitions of the motif and excluding $(\mathrm{CG})_{\mathrm{n}}$ and $(\mathrm{AT})_{\mathrm{n}}$. To maximize the chances to obtain statistically unlinked loci, we excluded microsatellite loci belonging to the same D. suzukii scaffold and blasted the selected sequences to the Drosophila melanogaster (Meigen) genome to infer chromosomal location. We used the program PRIMER DESIGNER (version 2.0; Scientific \& Educational Software 1990/1991) to design forward and reverse primers for the chosen microsatellite loci.

Each pair of primers was used for PCR amplification in a $10 \mu \mathrm{L}$ final volume containing 1 X QIAGEN Multiplex Master Mix, $2 \mu \mathrm{M}$ of each primer, and $2 \mu \mathrm{L}$ of genomic DNA. PCR programs were set with an initial period of denaturation at $94^{\circ} \mathrm{C}(30 \mathrm{~s})$ followed by 32 cycles of additional denaturation at $94^{\circ} \mathrm{C}(30 \mathrm{~s})$, an annealing phase at $57^{\circ} \mathrm{C}(1 \mathrm{~min} 30 \mathrm{~s})$, an elongation phase at $72^{\circ} \mathrm{C}$ ( $1 \mathrm{~min})$, and ending with another extension phase at $72^{\circ} \mathrm{C}(30$ $\min )$.

We first used $1.5 \%$ agarose gel for migration of the PCR products. Loci for which amplifications produced only one DNA band corresponding to the predicted size of the PCR product were then amplified following the protocol described above but with forward primers fluorophore-labelled on the 5' end and non-labelled reverse primers. These loci were finally sized using an ABI 3730 sequencer (Applied Biosystems, Montpellier, France) with the $500 \mathrm{LIZ}^{\mathrm{TM}}$ GeneScan ${ }^{\mathrm{TM}}$ size standard and scored with the GENEMAPPER $^{\mathrm{TM}} 5.0$ software.

\section{Genetic analyses}

All loci were checked for null-alleles using FreeNa (Chapuis \& Estoup, 2007; Chapuis et al., 2008) and MicroChecker (van Oosterhout et al., 2004) in the Hawaii and Montpellier populations.

Levels of polymorphism and departure from Hardy-Weinberg (H-W) equilibrium were assessed for both populations. Linkage disequilibrium (LD) was tested for each pair of loci using GENEPOP 4.2 (Raymond \& Rousset, 1995). $F_{S T}$ values were estimated using both GENEPOP 4.2 and FreeNa (with the ENA correction for null alleles implemented in the program). To test for loci under selection, we used the DetSel package (Vitalis et al., 2012) for R (R Development Core Team, 2008). DetSel identifies markers showing deviation from the neutral expectation in pairwise comparisons of diverging populations (Vitalis et al., 2001). We used the following parameters for our analysis (details about the user's parameter options can be found in Vitalis et al., 2012): number of simulation points $=1.10^{6}$, mutation rate $=5.10^{-5}$, time of divergence $=5000$ and effective size $\left(N_{e}\right)=500,000$. We used a personal program to construct a Neighbor-Joining (NJ) dendrogram from individual genotypes using a variant of Chakraborty and Jin's allele-shared distance defined in Fournier et al. (2005).
We studied cross-species PCR amplification of our set of microsatellite loci, using the same PCR conditions as above, on individuals from four species belonging to the suzukii subgroup: $D$. biarmipes (Malloch), D. subpulchrella (Takamori \& Watabe), D. lucipennis (Lin) and D. mimetica (Bock \& Wheeler). Each sisterspecies was represented by a single isofemale line from which 12 individuals were genotyped (samples obtained from the UC San Diego Drosophila stock center).

\section{RESULTS}

A large number of regions containing continuous microsatellites located on different scaffolds of the D. suzukii genome assembly were identified using the QDD software. We ensured that the chosen loci were not aggregated on the D. suzukii genome by first BLAST searching them using the $D$. melanogaster genome. We then chose 32 loci matching two autosomal chromosomes (the third autosomal chromosome is of very small size in Drosophila species) and different regions within each chromosome, with a final distribution of 8 loci per chromosome arm. Thirty two pairs of primers, delimiting the 32 chosen loci, were designed for PCR amplifications. Twenty eight such markers were robustly sized and genotyped with GENEMAPPER ${ }^{\mathrm{TM}} 5.0$ and used for further analyses.

\section{Genetic diversity}

Table 1 shows that all 28 microsatellite loci were polymorphic with allele numbers ranging from 2 (DS19, DS34, DS11) to 11 (DS32) in the pooled dataset (France + Hawaii). Observed heterozygosity $(\mathrm{Ho})$ ranged from 0.19 (DS21) to 0.84 (DS14) in the Hawaii population and 0.12 (DS21) to 0.88 (DS05) in the French population. Only one locus (DS21) showed significant departure $(P<0.05)$ from $\mathrm{H}-\mathrm{W}$ equilibrium in the Hawaii population and two loci (DS21 and DS25) in the French population. Significant LD was found for one pair of loci in the Montpellier population and for 14 pairs in the Hawaii population. All pairs of loci presenting significant LD were putatively located on the same chromosome arm. Only one pair (DS38-DS39) showed significant LD in both populations.

\section{Testing for null alleles and selection}

FreeNa pointed to five loci with null allele frequencies $>10 \%$ in at least one population (DS09, DS25, DS06, DS26 and DS21). The same loci were identified as bearing null alleles in at least one population by Microchecker. Of these five loci, only one (DS21) showed high null allele frequencies in both populations and was therefore left out when testing for selection. DetSel did not detect any microsatellite locus that departed significantly from neutral expectations.

\section{Population differentiation}

We found a significant and substantial level of genetic differentiation between the populations from France and Hawaii $\left(\mathrm{F}_{\mathrm{ST}}\right.$ $=0.206$ ). In agreement with this, the $\mathrm{NJ}$ tree using individuals as units shows a clear clustering of individuals in relation to their geographic origin (Fig. 1). Moreover genetic assignment tests significantly assigned all genotyped individuals to their population of origin (results not shown).

\section{Cross-species PCR amplification}

Table 2 shows that most loci (24 out of 28) successfully amplified in both $D$. biarmipes and D. subpulchrella. Although their profiles were generally similar to those observed with $D$. suzukii, most loci were homozygous and exhibited substantially different allele sizes compared to D. suzukii. Cross-amplification success was lower in D. mimetica and D. lucipennis (16 and 14 loci, respectively), which was expected according to the further ge- 
TABLE 1. Primer sequences and diversity index for 28 microsatellite loci in two invasive populations of Drosophila suzukii. A - allele number, $\mathrm{Ho}$ - observed heterozygosity; He - expected heterozygosity, $\mathrm{N}$ - number of sampled individuals, * - significant departure from H-W equilibrium $(P<0.05)$, D.mel. - position of homologous loci on D. melanogaster chromosomes.

\begin{tabular}{|c|c|c|c|c|c|c|c|c|c|c|c|}
\hline \multirow{2}{*}{ Locus } & \multirow{2}{*}{ Repeat } & \multirow{2}{*}{ D.mel. } & \multirow{2}{*}{ Primer sequence $5^{\prime} \rightarrow 3^{\prime}$} & \multirow{2}{*}{ Dye } & \multirow{2}{*}{ Size range } & \multicolumn{3}{|c|}{ Hawaii $(\mathrm{N}=33)$} & \multicolumn{3}{|c|}{ Montpellier $(\mathrm{N}=27)$} \\
\hline & & & & & & $\mathrm{A}$ & Ho & $\mathrm{He}$ & $\mathrm{A}$ & Ho & $\mathrm{He}$ \\
\hline$\overline{\mathrm{DS} 05}$ & (TG) 10 & Chr 2L & $\begin{array}{l}\text { AGGATAACGCGCAGCTTGAC } \\
\text { TATGGAAGCTGGCAAGCAGA }\end{array}$ & Ned & 260 & 6 & 0.8387 & 0.6967 & 4 & .8800 & 0.7584 \\
\hline DS06 & (TG)11 & Chr 2L & $\begin{array}{l}\text { CGGTTCGAGTGCTTGTTAGA } \\
\text { ACACGTGGAGGACACCTTC }\end{array}$ & Fam & $130-190$ & 4 & 0.6061 & 0.6056 & 3 & 0.3333 & 0.4856 \\
\hline DS07 & (CA)13 & Chr 2L & $\begin{array}{l}\text { AAGGCTGGAGTGGCAACAA } \\
\text { GCTAAGGTTCTGTTCGGCTG }\end{array}$ & Ned & $160-210$ & 3 & 0.4545 & 0.5147 & 7 & 0.8148 & 0.8278 \\
\hline DS08 & $(\mathrm{AG}) 10$ & Chr 2L & $\begin{array}{l}\text { CGTTGTTGGCGGTGAGTAAG } \\
\text { GGCCATCAATCAGTCAGTCA }\end{array}$ & Vic & $110-170$ & 9 & 0.6970 & 0.7222 & 4 & 0.8148 & 0.7565 \\
\hline DS09 & (AC) 15 & Chr 2L & $\begin{array}{l}\text { CACACATGGCGTATGCGTAT } \\
\text { ACTTGTTGAGCCGTCCTGG }\end{array}$ & Fam & $190-250$ & 4 & 0.4848 & 0.6772 & 8 & 0.6538 & 0.6649 \\
\hline DS10 & (GT)11 & Chr 2L & $\begin{array}{l}\text { CGAGACTGTGCGAACGAGAG } \\
\text { CATATGCTGACTGCCTCACA }\end{array}$ & Ned & $270-330$ & 5 & 0.3939 & 0.5124 & 6 & 0.6538 & 0.6294 \\
\hline DS11 & (CA)11 & Chr 2L & $\begin{array}{l}\text { CGGTGACTCGTGCAGTTGTA } \\
\text { GCCGACTCTGTCTAGAGCAA }\end{array}$ & Vic & $230-290$ & 2 & 0.4545 & 0.3512 & 3 & 0.4074 & 0.4753 \\
\hline DS12 & (GT)19 & Chr 2R & $\begin{array}{l}\text { GCTGTTGCTGTTGCTATTGC } \\
\text { AGAACCGTTAGCTGAGCGAG }\end{array}$ & Fam & $320-380$ & 6 & 0.8438 & 0.7866 & 6 & 0.6538 & 0.7167 \\
\hline DS14 & (TG)10 & Chr 2R & $\begin{array}{l}\text { AAGAACCGCAACGAGCAA } \\
\text { GAATTATCCAGCGACACGAC }\end{array}$ & Fam & $180-220$ & 7 & 0.8485 & 0.7374 & 6 & 0.5833 & 0.5755 \\
\hline DS15 & (GT)11 & Chr 2R & $\begin{array}{l}\text { GGACAGCCGACATAAGAGG } \\
\text { GAGTTGCTGGCTCGACACTT }\end{array}$ & Fam & $260-320$ & 5 & 0.5152 & 0.5505 & 7 & 0.8462 & 0.6553 \\
\hline DS16 & (AC) 13 & Chr 2R & $\begin{array}{l}\text { TTCGTATGTTAGGCGCCA } \\
\text { CTGGCTGCTGACCTCAACTC }\end{array}$ & Pet & $100-140$ & 3 & 0.6061 & 0.6074 & 6 & 0.7308 & 0.7234 \\
\hline DS17 & (GT)10 & Chr 2R & $\begin{array}{l}\text { CATCTCAGGCCACGAATG } \\
\text { CTCCAGATTCTCGAGTGCAG }\end{array}$ & Fam & $80-130$ & 3 & 0.3939 & 0.3742 & 5 & 0.7407 & 0.7064 \\
\hline DS19 & (CT)10 & Chr 2R & $\begin{array}{l}\text { CCGTTGGCATCTCTGAGTCT } \\
\text { GCAGACGGAGAGCAGTTGTT }\end{array}$ & Vic & $180-220$ & 2 & 0.3636 & 0.3967 & 5 & 0.5556 & 0.5734 \\
\hline DS20 & (AG) 12 & Chr 3L & $\begin{array}{l}\text { CAGCCATATGCAATGCACTG } \\
\text { ATATCCAGCGGAAGTCGAGA }\end{array}$ & Ned & $210-270$ & 6 & 0.6250 & 0.6973 & 4 & 0.7083 & 0.7405 \\
\hline DS21* & (AC)11 & Chr 3L & $\begin{array}{l}\text { GAGACGCGATGGTACCGTTA } \\
\text { CCAATCGAGTGCAAGCGT }\end{array}$ & Vic & 0 & 4 & 0.1935 & $0.4886^{*}$ & 3 & 0.1176 & $0.5519^{*}$ \\
\hline DS22 & (GT)11 & Chr 3L & $\begin{array}{l}\text { TACAGATACGCCGTCGGATT } \\
\text { AAGACCAAGACGACGGACCT }\end{array}$ & Pet & $290-360$ & 6 & 0.5938 & 0.6948 & 5 & 0.6667 & 0.6979 \\
\hline DS23 & $(\mathrm{AC}) 10$ & Chr 3L & $\begin{array}{l}\text { TGCCACTAAGCTCACACGGT } \\
\text { CAGTTGCCACTTGCTGTGTA }\end{array}$ & Pet & $237-300$ & 6 & 0.6129 & 0.6145 & 3 & 0.7917 & 0.7283 \\
\hline DS25* & $(\mathrm{CA}) 10$ & Chr 3L & $\begin{array}{l}\text { CCTTCTGCTGTCCTGCTTAT } \\
\text { CGGTCGAGCATCAAGTGAA }\end{array}$ & Vic & -270 & 5 & 0.6667 & 0.6644 & 4 & 0.4444 & $0.7291 *$ \\
\hline DS26 & $(\mathrm{CA}) 10$ & Chr 3L & $\begin{array}{l}\text { CCTGTGTGCATCTCAGTGTT } \\
\text { TACAGCACTCCAGCACATGA }\end{array}$ & Vic & 60-130 & 4 & 0.3548 & 0.6113 & 4 & 0.7037 & 0.7277 \\
\hline DS27 & (GT)14 & Chr 3L & $\begin{array}{l}\text { CCAGCGACTGCAGAAGTGAC } \\
\text { GCAATCCTCCACAACACAAC }\end{array}$ & Ned & $80-130$ & 6 & 0.7576 & 0.7034 & 5 & 0.7037 & 0.6893 \\
\hline DS28 & (TG)11 & Chr 3L & $\begin{array}{l}\text { TTAAGCTGACCTCCTCCTCG } \\
\text { GCACTCGCACAGATACAAGG }\end{array}$ & Pet & $140-195$ & 4 & 0.5758 & 0.5822 & 7 & 0.8400 & 0.7896 \\
\hline DS32 & (TG)15 & Chr 3R & $\begin{array}{l}\text { CGGCGTGTTGCAGTTATTC } \\
\text { ATGCACTGGTCGACATGACA }\end{array}$ & Fam & $330-380$ & 4 & 0.8333 & 0.8073 & 10 & 0.7813 & 0.6685 \\
\hline DS33 & (AC) 10 & Chr 3R & $\begin{array}{l}\text { GTTGGATGCCTGGAGGAATA } \\
\text { TTGTGCGAGTATGTCAGCCA }\end{array}$ & Pet & $150-210$ & 3 & 0.6364 & 0.6318 & 5 & 0.8462 & 0.7655 \\
\hline DS34 & (GA)12 & Chr 3R & $\begin{array}{l}\text { AACAACGACGCAGAAGCTCA } \\
\text { CGACTGTTGCGCTCTGTAAT }\end{array}$ & Fam & $240-300$ & 5 & 0.4848 & 0.4444 & 2 & 0.5417 & 0.5634 \\
\hline DS35 & $(\mathrm{CA}) 11$ & Chr 3R & $\begin{array}{l}\text { TCCGTATTCCGTATCCGTGT } \\
\text { GGAGTATGGCAGTGTGGCAG }\end{array}$ & Pet & $198-240$ & 8 & 0.4688 & 0.5171 & 3 & 0.8333 & 0.7925 \\
\hline DS36 & (GT)13 & Chr 3R & $\begin{array}{l}\text { TTGGCAACGTGTGAAGCTG } \\
\text { GAGACACTGCAATGCTGCCT }\end{array}$ & Vic & -220 & 4 & 0.7879 & 0.7199 & 4 & 0.4074 & 0.5158 \\
\hline DS38 & $(\mathrm{AC}) 12$ & Chr 3R & $\begin{array}{l}\text { CTGTCGGCTACGCAATATTC } \\
\text { CAACGGATTGTGGCTGATTG }\end{array}$ & Ned & $130-180$ & 7 & 0.5758 & 0.4835 & 3 & 0.8148 & 0.7833 \\
\hline DS39 & $(\mathrm{AC}) 12$ & Chr 3R & $\begin{array}{l}\text { GCCAGGCAGCAACAACATC } \\
\text { AGATCCGAGAGTTGCGAGTT }\end{array}$ & Ned & $310-370$ & 6 & 0.7188 & 0.7466 & 5 & 0.7407 & 0.7257 \\
\hline
\end{tabular}




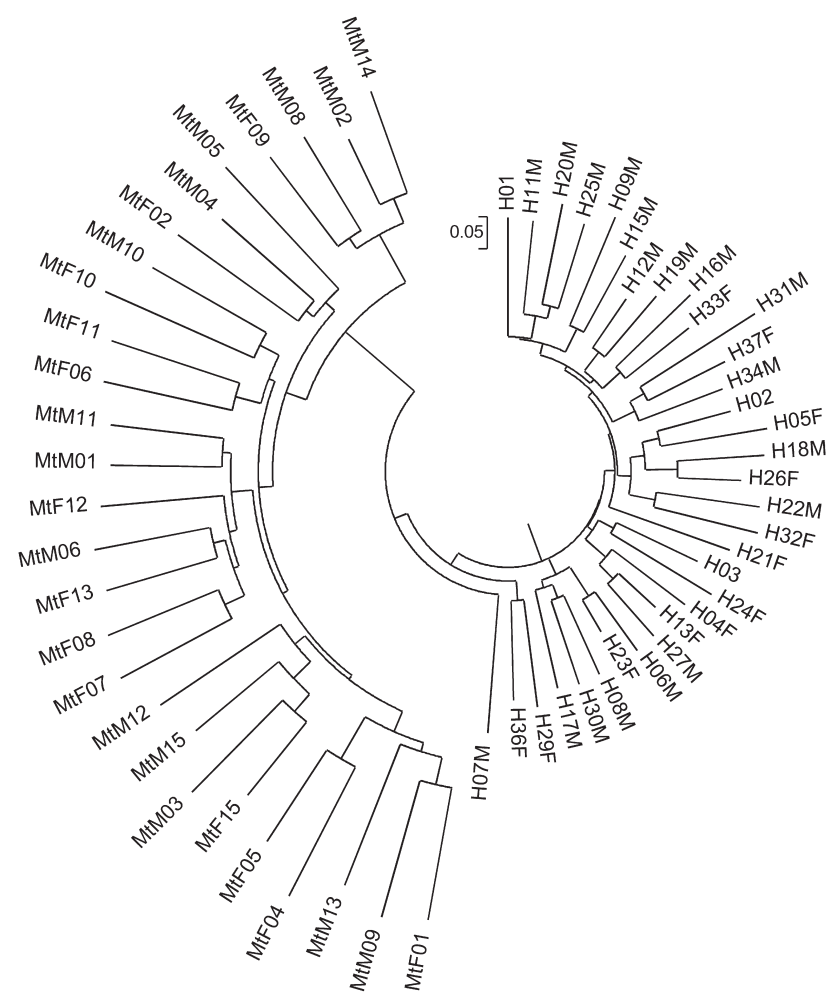

Fig. 1. NJ dendrogram of the microsatellite allele shared distances between $D$. suzukii individuals. Mt - French population; $\mathrm{H}$ - Hawaii population; $\mathrm{M} / \mathrm{F}$ - male or female fly (when known). netic distance of the two species from $D$. suzukii compared to $D$. biarmipes and D. subpulchrella (Kopp \& True, 2002). Because the individuals used for cross-amplification tests originated from stock-center populations (with a single isofemale line for each species), a high level of inbreeding and adaptation to laboratory conditions is suspected for these flies, thereby questioning the reliability of the observed allelic pattern in the non-suzukii species.

\section{DISCUSSION}

The inferential methods used for retracing invasion routes necessitates that the studied populations display substantial polymorphism and significant genetic differentiation in order to discriminate among potential origins and make inferences about demographic processes (e.g. Estoup \& Guillemaud, 2010). The microsatellite markers here developed for D. suzukii clearly fit these criteria, at least for the two tested populations. In a recent paper, Adrion et al. (2014) argued that at least $10^{5}$ independent sequence loci would be needed to confidently discriminate among colonization histories in D. suzukii using ABC methods. We believe that this pessimistic conclusion should be taken cautiously due to potential methodological issues: (i) the estimation of such $\mathrm{ABC}$ power relies on the analysis of pseudo-observed datasets simulated by drawing parameters into prior distributions, hence not conditionally on the observed dataset, and (ii) the number of sequence loci needed to attain $90 \%$ power $\left(10^{5}\right.$ loci) derives from a delicate extrapolation exercise based on pseudoobserved datasets simulated with a particularly small number of loci (comprising between 6 and 192 sequence loci, depending on the simulations). Interestingly, the same authors underline the fact that polymorphic markers such as microsatellites should better

TABLE 2. Cross-species amplification results for 28 D. suzukii microsatellite loci in four species of the suzukii subgroup. Cell values indicate allele numbers and size range (in brackets). $\mathrm{N}$ - number of individuals genotyped per species.

\begin{tabular}{|c|c|c|c|c|}
\hline$\overline{\text { Locus }}$ & D. biarmipes $(\mathrm{N}=12)$ & D. subpulchrella $(\mathrm{N}=12)$ & D. mimetica $(\mathrm{N}=12)$ & D. lucipennis $(\mathrm{N}=12)$ \\
\hline DS05 & $3(276-282)$ & $3(276-282)$ & - & - \\
\hline DS06 & $3(154-189)$ & $3(154-189)$ & $1(165)$ & $1(201)$ \\
\hline DS07 & $2(179-181)$ & $2(179-181)$ & $1(177)$ & - \\
\hline DS08 & - & - & $1(128)$ & $2(140-157)$ \\
\hline DS09 & $1(211)$ & $1(211)$ & $2(225-231)$ & $1(244)$ \\
\hline DS10 & $2(292-302)$ & $1(292)$ & - & - \\
\hline DS11 & $1(251)$ & $2(251-253)$ & $1(239)$ & $1(256)$ \\
\hline DS12 & $2(326-338)$ & $2(326-338)$ & - & - \\
\hline DS14 & $2(189-195)$ & $4(189-207)$ & $1(200)$ & $1(201)$ \\
\hline DS15 & $2(260-270)$ & $2(260-270)$ & $2(316-323)$ & $1(244)$ \\
\hline DS16 & $1(111)$ & $1(111)$ & $1(119)$ & $1(100)$ \\
\hline DS17 & $1(99)$ & $1(99)$ & - & - \\
\hline DS19 & $1(139)$ & $1(139)$ & - & - \\
\hline DS20 & $3(232-236)$ & $1(236)$ & $1(243)$ & - \\
\hline DS21 & - & - & - & - \\
\hline DS22 & $1(329)$ & $1(329)$ & $1(330)$ & $1(317)$ \\
\hline DS23 & $1(256)$ & $1(256)$ & - & $2(241-271)$ \\
\hline DS25 & $1(241)$ & $1(241)$ & $2(225-231)$ & - \\
\hline DS26 & $2(82-89)$ & $2(83-89)$ & - & - \\
\hline DS27 & $2(84-98)$ & $2(84-98)$ & $1(93)$ & $2(85-87)$ \\
\hline DS28 & $2(149-178)$ & $2(149-178)$ & - & $1(159)$ \\
\hline DS32 & $2(335-337)$ & $1(335)$ & $1(335)$ & - \\
\hline DS33 & $2(159-165)$ & $2(159-165)$ & - & - \\
\hline DS34 & $2(253-257)$ & $3(251-255)$ & $1(257)$ & $1(268)$ \\
\hline DS35 & - & - & - & - \\
\hline DS36 & $2(175-182)$ & $2(175-182)$ & $1(177)$ & $1(201)$ \\
\hline DS38 & $4(133-148)$ & $4(133-148)$ & $2(126-128)$ & $2(113-119)$ \\
\hline DS39 & $2(311-334)$ & $2(311-334)$ & - & - \\
\hline
\end{tabular}


discriminate colonization models and that additional $D$. suzuki population samples were needed to better discriminate such models. In agreement with this view, microsatellite markers (often analyzed using $\mathrm{ABC}$ methods) have proven invaluable for reconstructing invasion routes in many previous studies dealing with various biological models, and most of such studies were based on a larger set of population samples than those used in Adrion et al. (2014) (see for instance Lombaert et al., 2011 and 2014, and references therein). It is also worth noting that a lower number of microsatellite markers (compared to the set of 28 microsatellites we developed for $D$. suzukii) were used in many of these other studies. We hence believe that our suite of 28 markers represents a molecular tool with high potential when attempting to reconstruct the pathways and demographic processes of invasion in this major dipterous pest, in complement to the sequence data recently produce for $D$. suzukii populations as detailed in Adrion et al. (2014). We are currently using our suite of microsatellites to genotype a large number of population samples collected at various locations in the native and invasive range of $D$. suzukii to further refine the invasion history of this species on a worldwide scale.

ACKNOWLEDGEMENTS. This work benefited from state funding by the Agence Nationale de la Recherche, through the LabEx ANR-10-LABX-0003-BCDiv, of the program "Investissements d'avenir" (ref. ANR-11-IDEX-0004-02). Funding was also provided by the French Institute of Agricultural Research (INRASPE). We thank two anonymous referees and H.D. Loxdale for their helpful editorial comments.

\section{REFERENCES}

Adrion J.R., Kousathanas A., Pascual M., Burrack H.J., Haddad N.M., Bergland A.O., Machado H., Sackton T.B., Schlenke T.A., Watada M., Wegmann D. \& Singh N.D. 2014: Drosophila suzukii: the genetic footprint of a recent, worldwide invasion. - Mol. Biol. Evol. 31: 3148-3163.

Calabria G., Máca J., Bächli G., Serra L. \& Pascual M. 2012: First records of the potential pest species Drosophila suzukii (Diptera: Drosophilidae) in Europe. - J. Appl. Entomol. 136: 139-147.

Chapuis M.P. \& Estoup A. 2007: Microsatellite null alleles and estimation of population differentiation. - Mol. Biol. Evol. 24: 621-631.

Chapuis M.P., Lecoq M., Michalakis Y., Loiseau A., Sword G.A., PIRY S. \& Estoup A. 2008: Do outbreaks affect genetic population structure? A worldwide survey in Locusta migratoria, a pest plagued by microsatellite null alleles. - Mol. Ecol. 17: 3640-3653.

Chiu J.C., Jiang X., Zhao L., Hamm C.A., Cridland J.M., Saelao P., Hamby K.A., Lee E.K., KwoK R.S., Zhang G., Zalom F.G., Walton V.M. \& Begun D.J. 2013: Genome of Drosophila suzukii, the Spotted Wing Drosophila. - G3 Genes Genomes Genet. 3: 2257-2271.

Cini A., Anfora G., Escudero-Colomar L.A., Grassi A., Santosuosso U., Seljak G. \& PAPINI A. 2014: Tracking the invasion of the alien fruit pest Drosophila suzukii in Europe. - J. Pest Sci. 87: 559-566.

Estoup A. \& Guillemaud T. 2010: Reconstructing routes of invasion using genetic data: why, how and so what? - Mol. Ecol. 19: 4113-4130

Estoup A., Largiadèr C.R., Perrot E. \& Chourrout D. 1996 One-tube rapid DNA extraction for reliable PCR detection of fish polymorphic markers and transgenes. - Mol. Mar. Biol. Biotechnol. 5: 295-298.
Estoup A., Wilson I.J., Sullivan C., Cornuet J.M. \& Moritz C. 2001: Inferring population history from microsatellite and enzyme data in serially introduced cane toads, Bufo marinus. Genetics 159: 1671-1687.

Fournier D., Estoup A., Orivel J., Foucaud J., Jourdan H., Le Breton J. \& KelLer L. 2005: Clonal reproduction by males and females in the little fire ant. - Nature 435: 1230-1235.

Hauser M. 2011: A historic account of the invasion of Drosophila suzukii (Matsumura) (Diptera: Drosophilidae) in the continental United States, with remarks on their identification. - Pest Manag. Sci. — 67: 1352-1357.

KimURA M. 2004: Cold and heat tolerance of drosophilid flies with reference to their latitudinal distributions. - Oecologia 140: 442-449.

Kopp A. \& True J.R. 2002: Evolution of male sexual characters in the oriental Drosophila melanogaster species group. - Evol. Devel. 4: 278-291.

Lombaert E., Guillemaud T., Thomas C., Lawson Handley L.J., Li J., Wang S., Pang H., Goryacheva I.I., Zakharov I.A., Jousselin E., Poland R., Migeon A., van Lenteren J.C., De Clerce P., Berkvens N., Jones W. \& Estoup A. 2011: Inferring the origin of populations introduced from a genetically structured native range by approximate Bayesian computation: case study of the invasive ladybird Harmonia axyridis. - Mol. Ecol. 20: 4654-4670.

Lombaert E., Guillemaud T., Lundgren J., Koch R., Facon B., Grez A., Loomans A., Malausa T., Nedved O., Rhule E., Staverlokk A., Steenberg T. \& Estoup A. 2014: Complementarity of statistical treatments to reconstruct worldwide routes of invasion: the case of the Asian ladybird Harmonia axyridis. - Mol. Ecol. 23: 5931-6205.

Matsumura S. 1931: 6000 Illustrated Insects of Japan-Empire. Tokohshoin, Tokyo, 1497 pp. [in Japanese].

Meglécz E., Pech N., Gilles A., Dubut V., Hingamp P., Trilles A., GRENIER R. \& MARTIN J.F. 2014: QDD version 3.1: a userfriendly computer program for microsatellite selection and primer design revisited: experimental validation of variables determining genotyping success rate. - Mol. Ecol. Res. 14: 1302-1313.

PARShad R. \& PAIKA I.J. 1965: Drosophilid survey of India II. Taxonomy and cytology of the subgenus Sophophora (Drosophila). - Res. Bull. Panjab Univ. 5: 225-252.

RAYMOND M. \& RousSET F. 1995: GENEPOP (version 1.2): population genetics software for exact tests and ecumenicism. $-J$. Hered. 86: 248-249.

Thresher R.E., Hayes K., Bax N.J., Teem J., Benfey T.J. \& Gould F. 2014: Genetic control of invasive fish: technological options and its role in integrated pest management. - Biol. Invas. 16: $1201-1216$.

Van Oosterhout C., Hutchinson W.F., Wills D.P.M. \& Shipley P. 2004: Micro-checker: software for identifying and correcting genotyping errors in microsatellite data. - Mol. Ecol. Notes 4: $535-538$.

Vitalis R., Dawson K. \& Boursot P. 2001: Interpretation of variation across marker loci as evidence of selection. - Genetics 158: $1811-1823$.

Vitalis R. 2012: DetSel: a R-package to detect marker loci responding to selection. In Pompanon F. \& Bonin A. (eds): Data Production and Analysis in Population Genomics: Methods and Protocols. Springer/Humana Press, New York, pp. 277293.

Received December 3, 2014; revised and accepted April 21, 2015 Prepublished online July 21, 2015 